OPEN ACCESS

Edited by:

Marco Canevelli,

Sapienza Università di

Roma, Italy

Reviewed by:

Svetlana Ukraintseva,

Duke University, United States

Philipe De Souto Barreto,

Centre Hospitalier Universitaire

(CHU) de Toulouse, France

*Correspondence:

Maura Marcucci

marcucci.maura@gmail.com

Specialty section:

This article was submitted

to Geriatric Medicine,

a section of the journal

Frontiers in Medicine

Received: 08 September 2017 Accepted: 06 November 2017

Published: 22 November 2017

Citation:

Dolci GAM, Damanti S, Scortichini V,

Galli A, Rossi PD, Abbate $C$,

Arosio $B$, Mari $D$, Arighi $A$,

Fumagalli GG, Scarpini E, Inglese $S$

and Marcucci M (2017) Alzheimer's

Disease Diagnosis: Discrepancy

between Clinical, Neuroimaging,

and Cerebrospinal Fluid Biomarkers

Criteria in an Italian Cohort

of Geriatric Outpatients:

A Retrospective Crosssectional Study.

Front. Med. 4:203.

doi: 10.3389/fmed.2017.00203

\section{Alzheimer's Disease Diagnosis: Discrepancy between Clinical, Neuroimaging, and Cerebrospinal Fluid Biomarkers Criteria in an Italian Cohort of Geriatric Outpatients: A Retrospective Cross-sectional Study}

Giulia A. M. Dolci ${ }^{1,2}$, Sarah Damanti ${ }^{1,3}$, Valeria Scortichini ${ }^{1,4}$, Alessandro Galli, ${ }^{1,5}$, Paolo D. Rossi ${ }^{1}$, Carlo Abbate', Beatrice Arosio ${ }^{1,2}$, Daniela Mari ${ }^{1,2}$, Andrea Arighi, Giorgio G. Fumagalli ${ }^{6,7}$, Elio Scarpini ${ }^{6}$, Silvia Inglese ${ }^{1}$ and Maura Marcucci ${ }^{1,8 *}$

\begin{abstract}
'Geriatric Unit, Fondazione IRCCS Ca' Granda Ospedale Maggiore Policlinico, Milan, Italy, ${ }^{2}$ Department of Clinical Sciences and Community Health, University of Milan, Milan, Italy, ${ }^{3}$ Nutritional Sciences, Università degli Studi di Milano, Milan, Italy, ${ }^{4}$ Geriatric Unit, Medical Department Maggiore Hospital, Bologna, Italy, ${ }^{5}$ Geriatric Unit, ASST Lariana, Ospedale Sant'Anna, Como, Italy, ${ }^{6}$ Neurodegenerative Disease Unit, Department of Pathophysiology and Transplantation, Centro Dino Ferrari, University of Milan, Fondazione IRCCS Ca' Granda Ospedale Maggiore Policlinico, Milan, Italy, ' Department of Neurosciences, Psychology, Drug Research and Child Health (NEUROFARBA), University of Florence, Florence, Italy, ${ }^{8}$ Department of Health Research Methods, Evidence, and Impact, McMaster University, Hamilton, ON, Canada
\end{abstract}

Background: The role of cerebrospinal fluid (CSF) biomarkers, and neuroimaging in the diagnostic process of Alzheimer's disease (AD) is not clear, in particular in the older patients.

Objective: The aim of this study was to compare the clinical diagnosis of AD with CSF biomarkers and with cerebrovascular damage at neuroimaging in a cohort of geriatric patients.

Methods: Retrospective analysis of medical records of $\geq 65$-year-old patients with cognitive impairment referred to an Italian geriatric outpatient clinic, for whom the CSF concentration of amyloid- $\beta(A \beta)$, total Tau (Tau), and phosphorylated Tau ( $p$-Tau) was available. Clinical diagnosis (no dementia, possible and probable AD) was based on the following two sets of criteria: (1) the Diagnostic Statistical Manual of Mental Disorders (DSM-IV) plus the National Institute of Neurological and Communicative Disorders and Stroke and the Alzheimer's Disease and Related Disorders Association (NINCDS-ADRDA) and (2) the National Institute on Aging-Alzheimer's Association (NIA-AA). The Fazekas visual scale was applied when a magnetic resonance imaging scan was available.

Results: We included 94 patients, mean age 77.7 years, mean Mini Mental State Examination score 23.9. The concordance (kappa coefficient) between the two sets of clinical criteria was $70 \%$. The mean CSF concentration $(\mathrm{pg} / \mathrm{ml})( \pm S D)$ of biomarkers was as follows: A 687 ( \pm 318$)$, Tau $492( \pm 515)$, and p-Tau $63( \pm 56)$. There was a trend for lower $A \beta$ and higher Tau levels from the no dementia to the probable AD group. The 
percentage of abnormal liquor according to the local cutoffs was still 15 and $21 \%$ in patients without AD based on the DSM-IV plus NINCDS-ADRDA or the NIA-AA criteria, respectively. The exclusion of patient in whom normotensive hydrocephalus was suspected did not change these findings. A total of $80 \%$ of patients had the neuroimaging report describing chronic cerebrovascular damage, while the Fazekas scale was positive in $45 \%$ of patients overall, in $1 / 2$ of no dementia or possible AD patients, and in about $1 / 3$ of probable AD patients, with no difference across ages.

Conclusion: We confirmed the expected discrepancy between different approaches to the diagnosis of $A D$ in a geriatric cohort of patients with cognitive impairment. Further research is needed to understand how to interpret this discrepancy and provide clinicians with practical guidelines.

Keywords: Alzheimer, aging, clinical criteria, biomarkers, neuropsychological tests, cerebrovascular disease

\section{INTRODUCTION}

For decades, Alzheimer's disease (AD) has been diagnosed only based on clinical criteria (1). With the increasing knowledge of the pathogenic processes underlying this and other dementias, several biomarkers have been proposed to support the diagnosis, also at early stages (2-4). Biomarkers are defined as any objective measurement of an in vivo pathological process (5). Cerebrospinal fluid (CSF) proteins [amyloid- $\beta$ (A $\beta$ ) protein, total Tau (Tau), and phosphorylated Tau (p-Tau)] and functional and anatomical neuroimaging findings represent the most studied biomarkers of AD.

The role of these biomarkers in the diagnosis of $\mathrm{AD}$ in clinical practice has not been completely clarified yet, as both the American (1) and International Working Group Guidelines (6) underline. Understanding their role in the diagnostic workflow might be particularly challenging in an older population ( $>85$ years) presenting with cognitive impairment. There is evidence suggesting that, as age increases, the prevalence of pathological patterns that have been associated with the disease, increases also in subjects without cognitive impairment $(7,8)$. Also, the association between the presence of neuritic plaques in autoptic specimens and dementia is less strong in older people $(9,10)$. Changes in neuroimaging may be less salient in the older ages, in which atrophy often coexists with cerebrovascular damage. Furthermore, chronic cerebrovascular disease is such a frequent neuroimaging finding that its contribution to the cognitive deficit remains difficult to define, especially when not properly quantified (11). Finally, in the oldest patients, the burden of comorbidities often makes the scenario more complex (6). All these reasons increase the chance of conflicting findings between biomarkers and clinical symptoms. The whole picture is further complicated by the fact that the existing sets of diagnostic criteria for $\mathrm{AD}$ proposed by different scientific societies assign a different place to some clinical symptoms and signs. In fact, the diagnostic criteria have changed over time, integrating the new knowledge upon the disease mechanisms and biomarkers and reflecting different disease definitions $(1,6,12)$. However, the newer criteria have not replaced the older ones, which are still being used in clinical research, and in particular in studies evaluating therapies for $\mathrm{AD}$ (13). The dimension of the problem can be substantial and represents a barrier to a straightforward diagnostic process in routine practice, especially in those clinical settings providing care to less selected older patients such as geriatrics.

With such a background, the objective of our study was to represent the level of discrepancy between different diagnostic approaches, describing a population of older patients with cognitive impairment referred to an Italian geriatric outpatient clinic. In particular, we compared the diagnosis based on clinical criteria with the CSF biomarkers and with cerebrovascular damage finding at neuroimaging.

\section{MATERIALS AND METHODS}

\section{Study Design and Population}

This is a retrospective cross-sectional study of medical records of 65-year-old or older patients, referred to the Alzheimer Evaluation Unit (UVA) of the Division of Geriatrics of the IRCCS Ca' Granda Ospedale Maggiore Policlinico in Milan between June 2009 and October 2014. Ethical approval was not required for this study in accordance with the institutional guidelines.

We included in the study all those patients with a cognitive impairment who underwent at physician's discretion a lumbar puncture during the diagnostic workup and for whom the concentration of $\mathrm{A} \beta$, Tau, and $\mathrm{p}$-Tau in the CSF was available. There was no exclusion criterion. In particular, as per our practice, patients undergo a lumbar puncture with liquor collection and examination: (i) in the context of differential diagnosis of dementia, when the treating physician deems it as necessary to help confirm or rule out a clinical suspicion of $\mathrm{AD}$, and (ii) in the context of diagnosis and therapy (i.e., ex juvantibus) of normotensive hydrocephalus.

All patients undergo the lumbar puncture only if a specific written informed consent was provided by the patient or by her/his next of kin.

Retrospectively, but in a blind fashion with respect to the actual diagnosis made by the treating physician, we characterized 
the patients according to different diagnostic approaches. We first classified the patients using two different sets of clinical diagnostic criteria for $\mathrm{AD}$ (not taking into account the laboratory findings) and then compared the clinical diagnoses with the results of CSF biomarkers. Second, we described the prevalence of signs of vascular damage at neuroimaging according to different approaches, i.e., standard descriptive reports versus visual quantitative scales, and its correlation with the clinical and the liquor-based classifications. Within this framework, we also evaluated the contribution of neuropsychological (NPS) tests in making the diagnosis of $\mathrm{AD}$. In fact, it is known that many patients with cognitive impairment have poor awareness or understanding of their cognitive impairment (14). Thus, an objective cognitive assessment lies at the core of an appropriate diagnostic workup for cognitive decline. Moreover, NPS tests can help define early or prodromal states like a mild cognitive impairment $(\mathrm{MCI})$, in which biomarkers might be already positive $(6,15,16)$. Finally, all the patients for whom a brain magnetic resonance imaging scan was available were included in the sub-study on neuroimaging.

To have an objective comparison across different diagnostic tools, we included all patients with a CSF record, regardless of the final diagnosis made by the treating physician. Patients with a clinical suspicion of normotensive hydrocephalus were included in the main analyses as expected negative cases (i.e., cases in which CSF biomarkers were expected to be negative). They were then excluded as a sensitivity analysis.

\section{Data Collection}

Patient medical records temporarily close but preceding the time of the lumbar puncture were evaluated for the purpose of our study. The study investigators were guarantor for protecting the confidential data from any inappropriate use beyond the purpose of this study.

\section{Clinical Diagnostic Criteria and NPS Assessment}

Two investigators (GD and AG) screened the patient charts independently and retrospectively reanalyzed medical records of patients included in the study, being blinded to the diagnosis that was made by the treating geriatrician. Clinical diagnosis of dementia and of AD was based on the criteria of the Diagnostic Statistical Manual of Mental Disorders (DSM-IV) (17) and of the National Institute of Neurological and Communicative Disorders and Stroke and the Alzheimer's Disease and Related Disorders Association (NINCDS-ADRDA) 1984 (18), respectively (Appendix in Supplementary Material). Patients were also classified according to the criteria for dementia and $\mathrm{AD}$ of the National Institute on Aging-Alzheimer's Association (NIA-AA) 2011 (1) (Appendix in Supplementary Material). Based on each of the two sets of clinical criteria, patients were classified into no dementia, possible $\mathrm{AD}$, and probable $\mathrm{AD}$. The results of the following clinical investigations were taken into account for the classification of each patient upon those criteria, when available: the multidimensional geriatric assessment, blood tests, NPS tests, and neuroimaging. In particular, we relied on the results of the NPS assessment, when available, in case of inconsistency between the NPS report and the record of the geriatric visit for what concerned the presence of memory deficits and the level of impact on function. In order to preserve the comparative analyses of our sub-study, when we applied the clinical diagnostic criteria, we used the neuroimaging reports only to rule out the presence of a clear alternative diagnosis (i.e., normotensive hydrocephalus, multi-infarct disease, and tumor). The descriptive finding of "leukoaraiosis" or "chronic cerebrovascular disease" was not considered sufficient for meeting the criterion of an alternative (i.e., vascular) etiology of dementia.

We looked for the NPS assessment that preceded or coincided with the date of the lumbar puncture. In patients with multiple assessments, we used the outcome of the assessment that was temporarily closer to the date of the lumbar puncture. We used the outcome of NPS tests performed after the lumbur puncture only if temporarily very close (no more than 1 month later). The battery of NPS tests was administered by an expert neuropsychologist. Global cognitive functioning was assessed by means of the Mini Mental State Examination (MMSE) (19) and general intellectual functioning was investigated by using Raven's colored progressive matrices (20). For temporal orientation, the first item of the MMSE was considered. Anterograde long-term memory was rated with the prose recall test (21) and the delayed recall of the Rey-Osterreith complex figure test (17). Verbal short-term memory was assessed by means of the forward digit span test (18). The digit cancelation test (21) was administered to examine visual attention. Executive prefrontal functions were evaluated using the backward digit span test (17), the trail-making test (22), and the phonological fluency test (23). Spatial skills were divided into spatial orientation, assessed by the second item of the MMSE, and spatial abilities, explored by means of the copy of geometrical figure test (20) and the copy of the Rey-Osterreith complex figure test (17). Language was examined using the picture-naming test (24). All tests, excepted for the orientation one, have been validated and standardized in a sample of healthy Italian subjects. Most of the normative data are referred to the study from Spinnler and Tognoni (21). According to the outcome of the NPS assessment, patients were classified into: normal cognition, MCI, or diffuse/severe cognitive impairment. Patients classified as MCI were divided into the following four subtypes: only memory domain affected, singledomain MCI with a deficit other than on memory domain, multiple domain MCI with memory domain affected, and multiple domain MCI with deficits other than on memory domain.

\section{CSF Biomarkers}

The lumbar puncture was performed according to procedural standards. The dosage of A $\beta$ protein, Tau, and $\mathrm{p}$-Tau in the liquor was performed on site. The cerebrospinal fluid sample was centrifuged at $4^{\circ} \mathrm{C}$ and stored at $-30^{\circ} \mathrm{C}$ until analysis. A $\beta 42$ protein, Tau and p-Tau 181 were determined by ELISA kits (Innogenetics). The local laboratory cutoff points for normal protein concentrations are as follows: A $\beta 42>600 \mathrm{pg} / \mathrm{ml}$, Tau $<500 \mathrm{pg} / \mathrm{ml}$, and $\mathrm{p}$-Tau $<61 \mathrm{pg} / \mathrm{ml}$.

\section{Neuroimaging}

Available brain MRI images were examined independently and retrospectively by three operators blinded to the patient clinical 
history. The Fazekas visual scale (25) was applied on at least one long TR sequence, Flair or T2. Given the smallest number of missing data, for the purpose of the analysis, only axial plane images were considered. The Fazekas scores range from 0 (normal) to 3 (extensive, diffuse, and confluent lesions of the subcortical white matter). For the purpose of our analyses, we dichotomized the Fazekas scores into negative ( 0 or 1$)$ and positive $(\geq 2)$. We chose this cutoff in order to be more specific in this population at high prevalence of chronic cerebrovascular damage.

We decided not to include the assessment of atrophy according to the qualitative versus quantitative approach in our comparative analyses, because only a very small subgroup of patients had suitable MRI images to apply atrophy quantitative scales. Functional neuroimaging (positron emission tomography) was available only for few patients.

\section{Statistical Analysis}

Descriptive statistics (mean, SD, median and range in case of numerical variables, and frequency in case of categorical variables) were used to present the classification of patients according to the clinical criteria (DSM-IV plus NINCDS-ADRDA versus NIA-AA), the CSF biomarkers, and the neuroimaging biomarkers of cerebrovascular disease. First, the two different clinical criteria were compared and the concordance was measured by Cohen's kappa calculation (to take into account the effect of chance). Distributions of biomarkers were compared with the clinical diagnoses according to the NIA-AA criteria, using cross tabulations and Pearson $\chi^{2}$ or Kruskal-Wallis test, in the whole cohort and by age groups. As sensitivity analyses, the comparison was repeated (i) excluding patients that underwent the lumber puncture in the context of a suspicion of normotensive hydrocephalus and (ii) taking into account the NPS diagnosis. Inter-rater reproducibility for the MRI visual scales was also calculated as Kappa.

\section{RESULTS}

The clinical records of 94 patients were examined. Table 1 shows the baseline characteristics of the study cohort. In most of the cases (68\%), the lumbar puncture was performed in the context of a differential diagnosis for AD. In 11 of these 64 patients, alternative dementia etiologies were considered: Lewi Body Dementia in four patients; Fronto-Temporal Dementia in six patients (in one of these patients normotensive hydrocephalus etiology was also under consideration); and subclinical hypothyroidism in one patient. In 30 patients, normotensive hydrocephalus was the main diagnostic hypothesis and the main reason for the lumbar puncture.

Table 2 summaries the availability of data for the comparison of the different diagnostic tools.

\section{Classifications According to Clinical Diagnostic Criteria and CSF Biomarkers}

A total of 55 (58\%), 13 (14\%), and 26 (28\%) patients were classified as being affected by no dementia, possible $\mathrm{AD}$, and probable $\mathrm{AD}$, respectively, according to the DSM-IV plus NINCDS-ADRDA criteria; 39 (41\%), 27 (29\%), and 28 (30\%), respectively, according
TABLE 1 | Baseline characteristics.

\begin{tabular}{lc}
\hline Characteristics & Distribution \\
\hline Mean age (SD), years & $77.7(5.2)$ \\
Female, $n$ (\%) & $58(61.7)$ \\
Mean MMSE (SD) & $23.9(4.1)$ \\
Mean basic ADL score (SD) & $4.7(1.6)^{\mathrm{a}}$ \\
Mean instrumental ADL score (SD) & $4.3(2.5)^{\mathrm{a}, \mathrm{b}}$ \\
History of hypertension, $n$ (\%) & $58(61.7)$ \\
History of diabetes mellitus, $n$ (\%) & $18(19.1)$ \\
History of dyslipidemia, $n$ (\%) & $40(42.5)$ \\
Smoker, $n$ (\%) & $53(53.4)$ \\
Yes & $10(10.6)$ \\
No & $31(33.0)$ \\
Ex & $9(9.6)$ \\
History of coronary artery disease, $n$ (\%) & $8(8.5)$ \\
History of stroke or TIA, $n$ (\%) & $4(4.2)$ \\
History of peripheral artery disease, $n$ (\%) & $43(45.7)^{\mathrm{c}}$ \\
Carotid atherosclerosis, $n$ (\%) & \\
\hline$n$, number; MMSE, Mini Mental State Examination; ADL, activity of daily living; & \\
TIA, transient ischemic attack. & \\
alnformation missing for one patient. &
\end{tabular}

TABLE 2 | Availability of data on the different diagnostic approaches in the study cohort.

Diagnostic approach

Number of patients with data (\% of the total cohort)

Clinical criteria

CSF biomarkers

94 (100)

NPS assessment

94 (100)

Neuroimaging -

$71(75)$

-standard repor

76 (81)

Neuroimaging -Fazekas scale

40 (42)

CSF, cerebral spinal Cerebrospinal fluid; NPS, neuropsychological.

TABLE 3 | Comparison of Diagnostic Statistical Manual of Mental Disorders (DSM-IV) plus National Institute of Neurological and Communicative Disorders and Stroke and the Alzheimer's Disease and Related Disorders Association (NINCDS-ADRDA) and National Institute on Aging-Alzheimer's Association (NIA$A A)$ criteria for the diagnosis of Alzheimer's disease (AD).

\begin{tabular}{|c|c|c|c|c|c|}
\hline \multirow{2}{*}{\multicolumn{2}{|c|}{$\begin{array}{l}\text { Clinical diagnostic } \\
\text { criteria }\end{array}$}} & \multicolumn{4}{|c|}{ NIA-AA criteria } \\
\hline & & $\begin{array}{c}\text { No } \\
\text { dementia, } \\
n(\%)\end{array}$ & $\begin{array}{c}\text { Possible AD, } \\
n(\%)\end{array}$ & $\begin{array}{l}\text { Probable } \\
\text { AD, } n(\%)\end{array}$ & $\begin{array}{l}\text { Total, } \\
n(\%)\end{array}$ \\
\hline \multirow{4}{*}{$\begin{array}{l}\text { DSM-IV plus } \\
\text { NINCDS- } \\
\text { ADRDA } \\
\text { criteria }\end{array}$} & $\begin{array}{l}\text { No dementia, } \\
n(\%)\end{array}$ & $39(71)$ & $16(29)$ & $0(0)$ & $55(58)$ \\
\hline & $\begin{array}{l}\text { Possible AD, } \\
n(\%)\end{array}$ & $0(0)$ & $11(85)$ & $2(15)$ & $13(14)$ \\
\hline & $\begin{array}{l}\text { Probable AD, } \\
n(\%)\end{array}$ & $0(0)$ & $0(0)$ & $26(100)$ & $26(28)$ \\
\hline & Total, $\boldsymbol{n}(\%)$ & $39(41)$ & 27 (29) & $28(30)$ & $94(100)$ \\
\hline
\end{tabular}

to the NIA-AA. As pre-specified, criterion on the presence of memory deficits was fulfilled using the objective outcome of the NPS assessment. In fact, $64 \%$ of those patients who had no objective memory deficit at the NPS tests had expressed a memory complaint during the clinical visit.

Table 3 compares patient classification according to the DSM-IV plus NINCDS-ADRDA with the NIA-AA clinical 
criteria. Every patient who was classified as demented according to the DSM-IV criteria was also classified as demented according to the NIA-AA criteria; whereas $29 \%$ classified as demented according to the NIA-AA criteria were not demented according to DSM-IV criteria. The crude concordance between the two sets of criteria for the diagnosis of dementia was $83 \%$, with a kappa coefficient of $67 \%$. The crude concordance for the specific diagnosis (no dementia, possible and probable AD) between the two criteria was $81 \%$ with a kappa of $70 \%$.

The mean (SD) value of $\mathrm{A} \beta$, Tau, and $\mathrm{p}$-Tau in the study population was $687 \mathrm{pg} / \mathrm{ml}$ (318), $492 \mathrm{pg} / \mathrm{ml}$ (515), and $63 \mathrm{pg} / \mathrm{ml}$ (56), respectively. According to the local laboratory cutoffs, $\mathrm{A} \beta$ and Tau values were on average normal whereas mean $\mathrm{p}$-Tau values were abnormal (high).

There was a statistically significant difference in the CSF concentration of $A \beta$ and Tau but not of $p$-Tau, across the three diagnoses made according to both NINCDS-ADRDA and NIA-AA criteria (Figure 1). In particular, there was a trend for lower $A \beta$ values and higher Tau levels going from the no dementia group to probable $\mathrm{AD}$ group, more evident in the case of the NINCDS-ADRDA diagnoses.

When the biomarkers levels were dichotomized based on local lab cutoffs into positive (i.e., abnormal) or negative (i.e., normal), the frequency of biomarkers positivity differed across the diagnoses in a statistically significant way only for A $\beta$, with both DSM-IV plus NINCDS-ADRDA and NIA-AA classification (4). Every biomarker tended to be more frequently positive in the case of patients with a diagnosis of probable $\mathrm{AD}$ compared to patients with a diagnosis of possible $\mathrm{AD}$ or no dementia (Table 4). Compared to patients with no dementia, patients with possible $\mathrm{AD}$ tended to present with positive biomarkers more frequently when DSM-IV plus NINCDSADRDA criteria were used but less frequently when NIA-AA criteria were used (Table 4).

Then, the CSF biomarkers were considered as a whole and the patient classified as having positive liquor only when the level of all the three proteins was abnormal (i.e., reduced $\mathrm{A} \beta$, elevated Tau, and p-Tau). In this case, only 18 patients (19\%) had positive liquor. Patients with positive liquor were on average younger than those with negative liquor [mean age $74.7(\mathrm{SD} \pm 3.7)$ versus 77.2 $(\mathrm{SD} \pm 2.7), p$ for Kruskal-Wallis test $=0.002$ ].

Table 5 shows the distribution of the liquor biomarker according to the different diagnoses and to different age groups. The trend for positive liquor was the same in the whole population and in the two age groups, with a higher prevalence of positive liquor in probable $\mathrm{AD}$ than in possible $\mathrm{AD}$ and no dementia, in both clinical classifications. The prevalence was again higher in those with no dementia than in those with possible dementia in the case of NIA-AA criteria. In any age group and in any clinical diagnosis group, a negative liquor was more prevalent than positive liquor (Table 5).

When we considered age cutoffs progressively lower than 80 , the percentage of patients with positive liquor became higher than the percentage of patients with negative liquor only among patients with a diagnosis of probable AD (any set of criteria) younger than 76 years (66\% positive versus $33 \%$ negative).
Thirty of the 94 patients underwent a lumbar puncture in the context of a clinical suspicion of normotensive hydrocephalus. When re-classified in a blinded fashion according to the two sets of clinical criteria, these patients were all classified as with no dementia according to the DSM-IV criteria. According to the NIA-AA criteria, 19 (63\%) patients were not demented and $11(27 \%)$ patients had a possible AD. Table 6 shows the comparison between the clinical (NIA-AA criteria) and the liquor diagnoses when patients with normotensive hydrocephalus were excluded. The trend did not change compared with the main analysis.

\section{NPS Assessment}

The outcome of the NPS assessment was available for 71 patients. Only one patient had a normal test performance; 44 patients were diagnosed as affected by diffuse cognitive impairment; and 26 patients were diagnosed as affected by MCI. Age was no significantly different between patients with diffuse cognitive impairment (mean 76.4 years, SD \pm 3.8 ) and patients with MCI (mean 76.4 years SD \pm 5.5 ). Twenty-six of the 27 patients classified as with no dementia according to the NIA-AA criteria (96.3\%) were diagnosed as affected by MCI, most of them (88\%) with deficits in multiple cognitive domains. In particular, the definition into MCI subtypes was available for 25 patients: three (12\%) patients were classified as single MCI with only memory domain affected; $15(60 \%)$ patients were classified as multiple domain MCI with memory domain affected; and seven (26\%) patients were classified as multiple domain MCI with deficits other than on memory domain. No patient was classified as single-domain MCI with a deficit other than on memory domain.

Tables $\mathbf{7}$ and $\mathbf{8}$ show the frequency of liquor positivity according to the NPS outcome and MCI phenotypes, respectively. There was no statistically significant difference in the frequency of positivity across NPS definitions. In particular, the biomarker liquor tended to be more frequently positive in patients with MCI than in patient with diffuse cognitive impairment.

\section{Cerebrovascular Burden at Neuroimaging}

MRI images were available for 40 patients. Thirty-two of these $40(80 \%)$ patients had a diagnosis of cerebrovascular damage according to the qualitative report made by the radiologist. Mean Fazekas score was $1.55 \pm 1$. According to the Fazekas score 18 of the 40 (45\%) patients were positive. Table 9 shows the mean Fazekas scores and positivity according to the clinical diagnostic criteria. According to both clinical classifications, Fazekas was positive in about half of the patients with no dementia or possible $\mathrm{AD}$, while it was positive in about onethird of the patients with probable AD. When only patients with a diagnosis of probable $\mathrm{AD}$ according to the NIA-AA criteria were considered, the proportion of patients with a positive Fazekas in progressively younger subgroups remained the same or increased compared with the whole population or with the oldest ones (Table 10). The results were the same for patients with probable $\mathrm{AD}$ according to DSM-IV plus NINCDS-ADRDA criteria. 

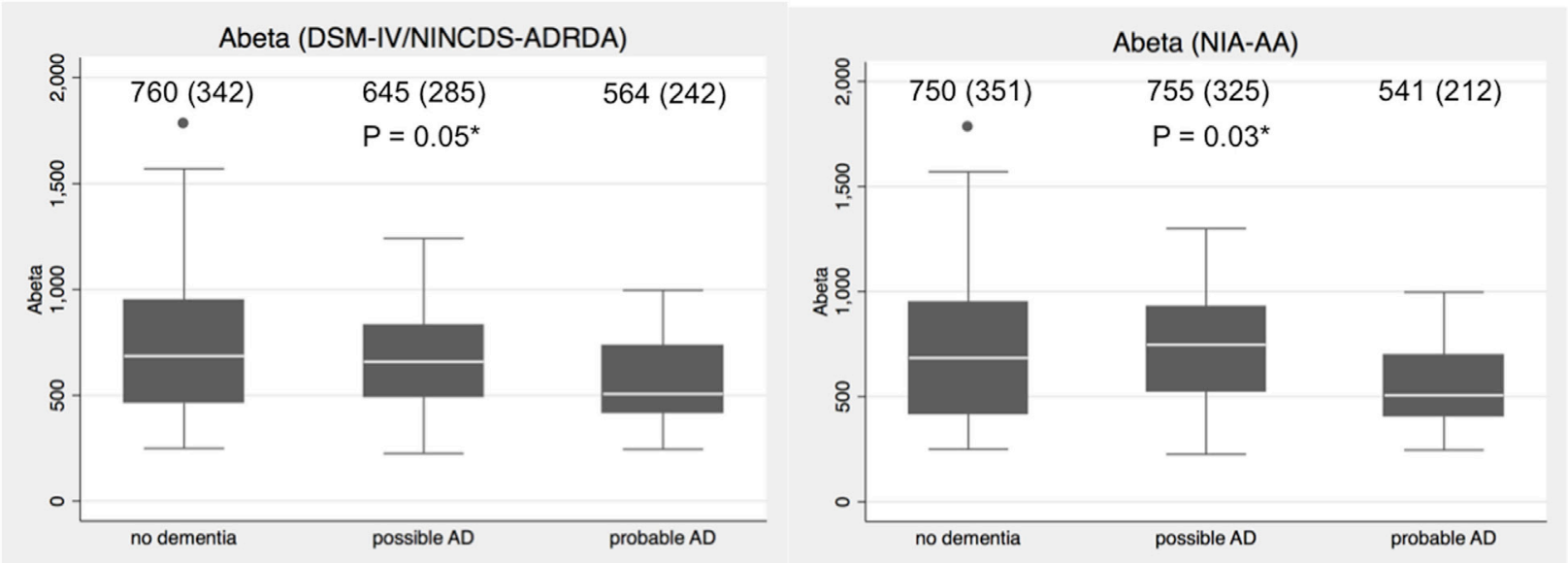

Tau (DSM-IV/NINCDS-ADRDA)
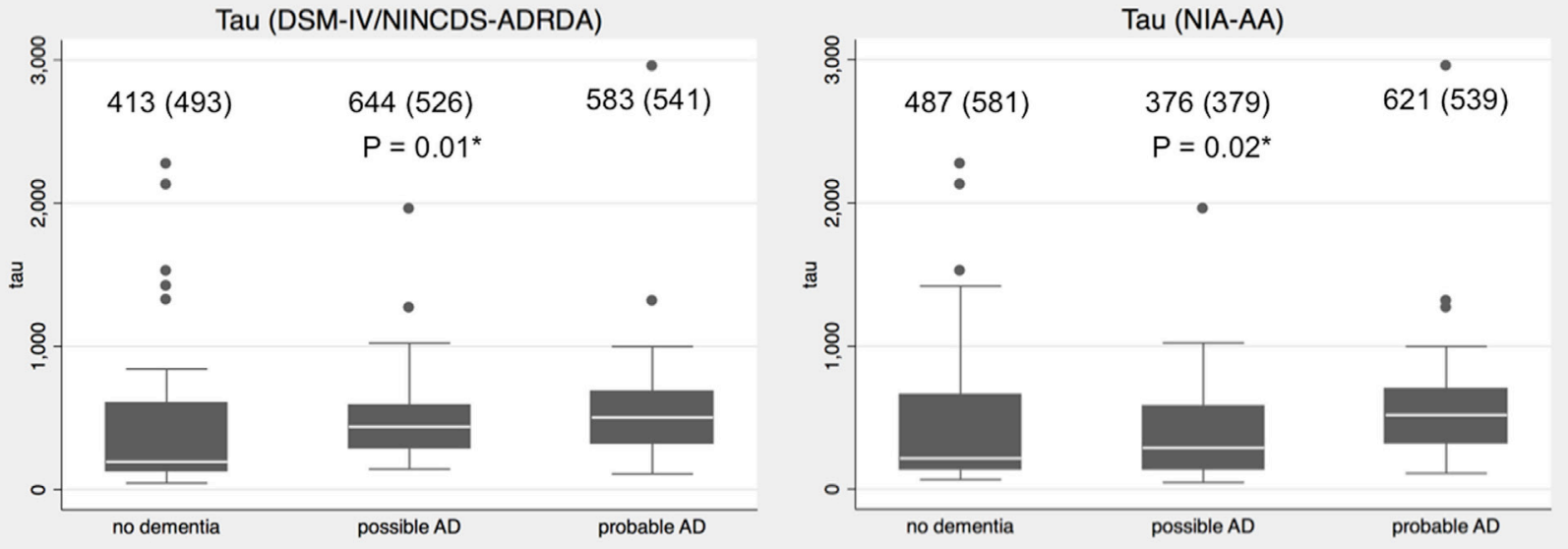

p-Tau (DSM-IV/NINCDS-ADRDA)
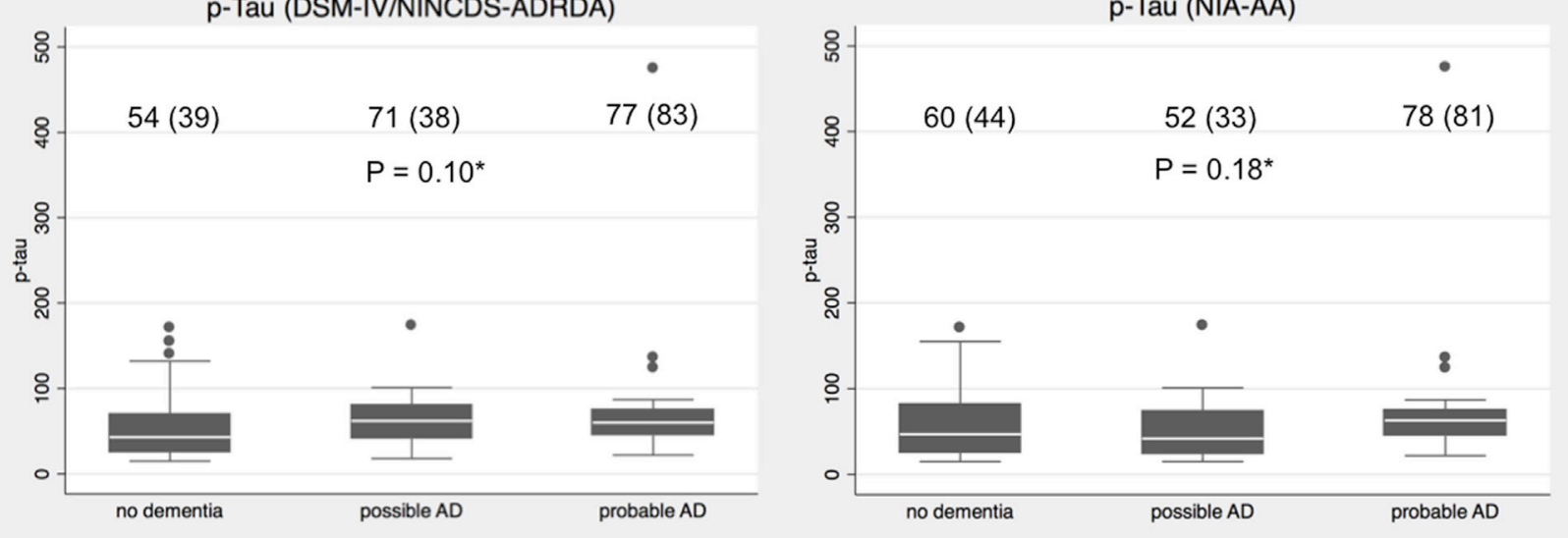

FIGURE 1 | Distributions of amyloid- $\beta$ (A $\beta$ ), total Tau (Tau), and phosphorylated Tau ( $p$-Tau) values in no dementia, possible Alzheimer's disease (AD) and probable AD patients according to Diagnostic Statistical Manual of Mental Disorders (DSM-IV) plus National Institute of Neurological and Communicative Disorders and Stroke and the Alzheimer's Disease and Related Disorders Association (NINCDS-ADRDA) and National Institute on Aging-Alzheimer's Association (NIA-AA) criteria. Legend: mean (SD) concentration is provided for each diagnostic category. *Kruskal-Wallis test for difference in the protein distribution across diagnostic groups.

When in the same patients with a clinical diagnosis of probable AD (according to NIA-AA or NINCDS-ADRDA criteria) the Fazekas results were cross-tabulated with the CSF results (Table 11), there was no statistically significant difference in frequency distribution.

\section{DISCUSSION}

Our retrospective analysis of a cohort of patients with a cognitive deficit referring to a geriatric outpatient clinic (mean age 78 years), confirmed a non-negligible discrepancy between the 
TABLE 4 | Relationship between CSF biomarkers and clinical diagnosis.

\begin{tabular}{|c|c|c|c|c|c|c|}
\hline & \multicolumn{3}{|c|}{$\begin{array}{l}\text { Diagnostic Statistical Manual of Mental Disorders-IV plus National } \\
\text { Institute of Neurological and Communicative Disorders and Stroke and } \\
\text { the Alzheimer's Disease and Related Disorders Association criteria }\end{array}$} & \multicolumn{3}{|c|}{$\begin{array}{c}\text { National Institute on Aging- Alzheimer's Association } \\
\text { criteria }\end{array}$} \\
\hline & Amyloid- $\beta(A \beta)$ & Total tau (Tau) & Phosphorylated tau (p-Tau) & $\mathbf{A} \boldsymbol{\beta}$ & Tau & p-Tau \\
\hline & Positive, $n$ (\%) & Positive, $n$ (\%) & Positive, $n$ (\%) & Positive, $n(\%)$ & Positive, $n(\%)$ & Positive, $n$ (\%) \\
\hline No dementia & $20(36)$ & $17(31)$ & 19 (35) & $16(41)$ & $12(31)$ & $15(39)$ \\
\hline Possible AD & $5(38)$ & $5(38)$ & 7 (54) & $8(30)$ & $8(30)$ & $9(33)$ \\
\hline Probable AD & $18(69)$ & $13(50)$ & $13(50)$ & $19(68)$ & $15(54)$ & $15(54)$ \\
\hline Pearson $\chi^{2}, p$ & 0.012 & 0.251 & 0.261 & 0.013 & 0.102 & 0.277 \\
\hline
\end{tabular}

TABLE 5 | Distribution of the biomarker liquor according to the Diagnostic Statistical Manual of Mental Disorders (DSM-IV) plus National Institute of Neurological and Communicative Disorders and Stroke and the Alzheimer's Disease and Related Disorders Association (NINCDS-ADDRDA) and National Institute on Aging-Alzheimer's Association (NIA-AA) diagnosis and age groups.

\begin{tabular}{|c|c|c|c|c|c|c|}
\hline \multirow[t]{2}{*}{ Age (years) } & \multicolumn{3}{|c|}{ DSM-IV plus NINCDS-ADRDA criteria } & \multicolumn{3}{|c|}{ NIA-AA criteria } \\
\hline & Diagnosis & Positive liquor, $\boldsymbol{n}(\%)$ & Pearson $\chi^{2}, p$ & Diagnosis & Positive liquor, $\boldsymbol{n}(\%)$ & Pearson $\chi^{2}, p$ \\
\hline \multirow[t]{3}{*}{ Any } & No dementia & $8(15)$ & 0.208 & No dementia & $8(21)$ & 0.027 \\
\hline & Possible AD & $2(15)$ & & Possible AD & $2(4)$ & \\
\hline & Probable AD & $8(31)$ & & Probable AD & 8 (32) & \\
\hline \multirow[t]{3}{*}{$\geq 80$} & No dementia & $2(7)$ & 0.324 & No dementia & $2(11)$ & 0.321 \\
\hline & Possible AD & $0(0)$ & & Possible AD & $0(0)$ & \\
\hline & Probable AD & $1(20)$ & & Probable AD & $1(20)$ & \\
\hline \multirow[t]{3}{*}{$<80$} & No dementia & $6(24)$ & 0.494 & No dementia & $6(27)$ & 0.168 \\
\hline & Possible AD & $2(16)$ & & Possible AD & $1(7)$ & \\
\hline & Probable AD & 7 (33) & & Probable AD & $8(35)$ & \\
\hline
\end{tabular}

TABLE 6 | Distribution of the biomarker liquor according to National Institute on Aging-Alzheimer's Association (NIA-AA) diagnosis in patients without a clinical suspicion of normotensive hydrocephalus.

\begin{tabular}{lcc}
\hline Diagnosis NIA-AA criteria & Positive liquor, $\boldsymbol{n}(\%)$ & Pearson $\boldsymbol{\chi}^{\mathbf{2}, \boldsymbol{p}}$ \\
\hline No dementia & $7(35)$ & 0.102 \\
Possible AD & $1(4)$ & \\
Probable AD & $9(32)$ & \\
Any & $17(27)$ & \\
\hline
\end{tabular}

TABLE 7 | Positivity of the biomarker according to different neuropsychological (NPS) diagnosis.

\begin{tabular}{lcc}
\hline & $\begin{array}{c}\text { Positive liquor among } \\
\text { all patients with NPS } \\
\text { assessment (71), } \boldsymbol{n} \text { (\%) }\end{array}$ & $\begin{array}{c}\text { Positive liquor among } \\
\text { those patients with NPS } \\
\text { assessment with no } \\
\text { normotensive hydrocephalus } \\
\text { suspect (62), } \boldsymbol{n} \text { (\%) }\end{array}$ \\
\hline $\begin{array}{l}\text { Cognitive normal } \\
\text { Mild cognitive }\end{array}$ & $1(100)$ & - \\
$\begin{array}{l}\text { impairment } \\
\text { iffuse cognitive }\end{array}$ & $7(27)$ & $7(35)$ \\
Total & $10(23)$ & $10(24)$ \\
\hline
\end{tabular}

diagnosis of $\mathrm{AD}$ when based on clinical criteria, CSF biomarkers, or neuroimaging.

First, we confirmed a substantial discordance between the two sets of clinical diagnostic criteria, i.e., DSM-IV plus
TABLE 8 | Positivity of the biomarker liquor according to different mild cognitive impairment (MCl) phenotypes and age groups.

\begin{tabular}{llcc}
\hline $\begin{array}{l}\text { Age } \\
\text { (years) }\end{array}$ & $\begin{array}{l}\text { Neuropsychological } \\
\text { phenotype }\end{array}$ & $\begin{array}{c}\text { Negative liquor, } \\
\boldsymbol{n}(\%)\end{array}$ & $\begin{array}{c}\text { Positive liquor, } \\
\boldsymbol{n}(\%)\end{array}$ \\
\hline$\geq 80$ & Amnestic MCl & 0 & 0 \\
& Multiple domain MCl+ & $3(60)$ & $2(40)$ \\
& Multiple domain MCl- & $5(100)$ & $0(0)$ \\
\hline$<80$ & Amnestic MCl & $2(67)$ & $1(33)$ \\
& Multiple domain MCl+ & $7(70)$ & $3(30)$ \\
& Multiple domain MCl- & $1(50)$ & $1(50)$ \\
\hline
\end{tabular}

+ , with amnestic component; -, without amnestic component.

NINCDS-ADRDA (1984) versus NIA-AA (2011) criteria, with an agreement of only $70 \%$ when adjusted for the effect of chance. The discordance likely reflects the evolution in the definition of dementia and AD and was someway expected. However, we wanted to quantify this discrepancy in a cohort of patients with a higher probability of a complex phenotype, since 1984 criteria have been used to define patient eligibility for approval studies of many current drugs available for $\mathrm{AD}$ and are still being used in research $(13,26-29)$. According to our data, most (16 out of $27,59 \%)$ of the "possible $\mathrm{AD}$ " patients according to the newer criteria would have been classified as "no dementia" by the older approach (Table 3) and would have been not eligible for those studies. The results of those studies are therefore not necessarily applicable to this subset of patients defined as "possible AD" according to a more comprehensive understanding of the disease. 
TABLE 9 | Cerebrovascular damage at neuroimaging according to the Fazekas scale and clinical diagnosis of dementia.

\begin{tabular}{|c|c|c|c|c|c|c|}
\hline \multirow[t]{2}{*}{ Diagnosis } & \multicolumn{3}{|c|}{$\begin{array}{l}\text { Diagnostic Statistical Manual of Mental Disorders-IV plus } \\
\text { National Institute of Neurological and Communicative Disorders } \\
\text { and Stroke and the Alzheimer's Disease and Related Disorders } \\
\text { Association criteria }\end{array}$} & \multicolumn{3}{|c|}{ National Institute on Aging-Alzheimer's Association criteria } \\
\hline & Fazekas, mean (SD) & $\begin{array}{c}\text { Positive Fazekas, }{ }^{\mathrm{a}} \\
\text { n (\%) }\end{array}$ & $p$ (Pearson $\chi^{2}$ test) & Fazekas, mean (SD) & $\begin{array}{c}\text { Positive Fazekas, }{ }^{\mathrm{a}} \\
n(\%)\end{array}$ & $p$ (Pearson $\chi^{2}$ test) \\
\hline No dementia & $1.1(1.1)$ & $12(52)$ & 0.453 & $1.6(1.1)$ & $10(53)$ & 0.451 \\
\hline Possible AD & $1.2(0.9)$ & $2(50)$ & & $1.6(1.1)$ & $4(50)$ & \\
\hline Probable AD & $1.4(1.0)$ & $4(31)$ & & $1.3(1.0)$ & $4(31)$ & \\
\hline
\end{tabular}

ascore $\geq 2$

TABLE 10 | Distribution of the positive Fazekas score in different age subgroups in people with a clinical diagnosis of probable AD (National Institute on AgingAlzheimer's Association criteria).

\begin{tabular}{lc}
\hline Age (years) & Positive Fazekas, $\boldsymbol{n}(\%)$ \\
\hline$\geq 80$ & $1(33)$ \\
$<80$ & $3(30)$ \\
$<75$ & $2(40)$ \\
$<72$ & $1(100)$ \\
\hline
\end{tabular}

TABLE 11 | Correlation of the biomarker liquor and cerebrovascular burden at neuroimaging according to Fazekas scores, in subjects with a clinical diagnosis of probable AD (National Institute on Aging-Alzheimer's Association criteria) in different age subgroups.

\begin{tabular}{lccc}
\hline & Negative Fazekas, $\boldsymbol{n}(\%)$ & Positive Fazekas, $\boldsymbol{n}$ (\%) & $\boldsymbol{p}$ \\
\hline $\begin{array}{l}\text { Total } \\
\text { Negative liquor }\end{array}$ & $6(67)$ & $3(33)$ & 0.764 \\
Positive liquor & $3(75)$ & $1(25)$ & \\
$<\mathbf{8 0}$ years & & & 0.778 \\
Negative liquor & $4(67)$ & $2(37)$ & - \\
Positive liquor & $3(75)$ & $1(25)$ & \\
$\mathbf{8} \mathbf{8 0}$ years & & & \\
Negative liquor & $2(67)$ & $1(33)$ & 0.709 \\
Positive liquor & $0(0)$ & $0(0)$ & \\
$\leq \mathbf{7 5}$ years & $1(50)$ & $1(50)$ & \\
Negative liquor & $2(67)$ & $1(33)$ & \\
Positive liquor & & & \\
$>\mathbf{7 5}$ years & $5(71)$ & $2(29)$ & \\
Negative liquor & $1(100)$ & $0(0)$ & \\
Positive liquor & & & \\
\hline
\end{tabular}

One of the main differences between the two criteria is that the older ones, but not the newer, include the presence of amnesic deficits as a necessary criterion for $\mathrm{AD}$ diagnosis. Interestingly, in our cohort, the majority of patients (64\%), who had no objective memory deficiency at the NPS tests, had in fact complained about forgetfulness during the clinical visit. This datum has been already described in the literature (30). This confirmed the role of the NPS assessment, which in practice might be sometimes forgone in the assessment of the oldest old patients. An extended NPS battery helped us to better define not only the phenotype but also the severity of the cognitive disorder $(6,31,32)$, which, in some cases, allowed us to suppose a higher functional impairment, or a higher contribution of the cognitive deficits to the functional impairment, among other possible health and social contributors, compared to what the interview with the patients or their caregivers had suggested.

The distribution of the CSF biomarkers levels in our population was quite sparse, even in patients with a clinically probable AD. In particular, patients with positive liquor biomarkers still represented a minority among those that would have been classified as probable AD based on clinical criteria only; they represented the majority only in a younger (i.e., <76 years) subset of patients. In 2012, Mattsson et al. investigated the effect of age on the diagnostic performance of CSF biomarkers in a large multi-center study population and they found that although the diagnostic accuracy for AD decreased with age, the predictive values for a combination of biomarkers remained essentially stable. In comparison with our population, their cross-sectional cohort of patients with $\mathrm{AD}$ had a lower median age (71 versus 77.7), an higher percentage of male subjects ( 57 versus $38.3 \%$ ) and a lower MMSE median score (22 versus 23.9) (33). In that study the clinical diagnosis of $\mathrm{AD}$ was based on $D S M$-IV plus NINCDS-ADRDA criteria. In our study too, there was a non-statistically significant trend for an increased liquor positivity going from "no dementia" to "possible" and then "probable AD," only when the DSM-IV plus NINCDSADRDA were used. This suggests a higher concordance between the CSF biomarkers so far known and the classical AD variant, rather than with the more comprehensive AD definition. In contrast, when we looked at the relationship between the CSF protein distribution and the NPS outcome, we did not find the expected association between a classical amnesic MCI phenotype and positive biomarkers.

Our findings confirm that quantitative methods based on neuroimaging (i.e., the Fazekas scale) can help refine the classification of patients upon the degree of cerebrovascular damage compared to descriptive radiological reports (34). Yet, the clinical relevance of neuroimaging remains uncertain among relatively older patients. Indeed, we less frequently found a positive Fazekas in patients with probable $\mathrm{AD}$, compared with patient with possible or no $\mathrm{AD}$, suggesting that the vascular damage is not a typical pathogenic mechanism of the disease. However, there was still a substantial percentage (31\%) of positive Fazekas among patients with probable AD. Furthermore, we found that a positive Fazekas tended to be only slightly more frequent among probable $\mathrm{AD}$ with negative liquor than among those with a positive liquor, regardless of age. This finally 
suggests that the two pathogenic pathways, i.e., the vascular and the degenerative, can definitely coexist and not necessarily only in patients who would easily meet the definition of vascular/mixed dementia (such as patients with a history of stroke).

The retrospective nature and the small sample size are the main limitations of our study, which could be only descriptive and explorative in nature. Then, although less selected in terms of age and clinical complexity than in randomized controlled trials, our cohort still represented a selected population. Indeed, including only patients who underwent a lumbar puncture might have led to the exclusion of the oldest and most complex patients for whom the lumbar puncture is more frequently thought not to have a favorable risk-benefit profile. Finally, we had to deal with missing and incomplete information, given the retrospective nature of our study and the use of data from routine practice. The time lag between the date in which the patient underwent the lumbar puncture and the time in which some other study variables were collected could represent a limitation to the actual concurrency of the cross-sectional comparison. However, this is consistent with the routine practice.

\section{CONCLUSION}

To conclude, we showed a significant degree of discordance between clinical criteria, NPS assessment, liquor biomarkers, and neuroimaging when used to characterize cognitive disorders in geriatric outpatients. Given the methodological limitations of our study, prospective larger multi-center studies, including inception cohorts of unselected patients that undergo a clinical, laboratory, and neuroradiological assessment, and with a clinical follow-up, would be theoretically necessary to better understand the role of biomarkers in the diagnostic workup of geriatric patients with cognitive disorders. However, practical and ethical issues might hinder the conduction of such a type of study, while the current demographic trend will lead quickly to a further increase of the prevalence of this patient population. Hence, researchers and clinicians in the field should make the efforts to combine their experience and expertise to reach a consensus on the best diagnostic practice in this population.

\section{REFERENCES}

1. McKhann GM, Knopman DS, Chertkow H, Hyman BT, Jack CR Jr, Kawas $\mathrm{CH}$, et al. The diagnosis of dementia due to Alzheimer's disease: recommendations from the National Institute on Aging-Alzheimer's Association workgroups on diagnostic guidelines for Alzheimer's disease. Alzheimers Dement (2011) 7(3):263-9. doi:10.1016/j.jalz.2011.03.005

2. Beach TG, Monsell SE, Phillips LE, Kukull W. Accuracy of the clinical diagnosis of Alzheimer disease at National Institute on Aging Alzheimer Disease Centers, 2005-2010. J Neuropathol Exp Neurol (2012) 71(4):266-73. doi:10.1097/NEN.0b013e31824b211b

3. Shaw LM, Vanderstichele H, Knapik-Czajka M, Clark CM, Aisen PS, Petersen RC, et al. Alzheimer's disease neuroimaging initiative. Cerebrospinal fluid biomarker signature in Alzheimer's disease neuroimaging initiative subjects. Ann Neurol (2009) 65(4):403-13. doi:10.1002/ana.21610

\section{ETHICS STATEMENT}

The study was a retrospective analyis of data routinely collected for clinical purpose, retrieved from patient medical charts. No approval from an ethics committee was sought. Given the nature of the study, the patients did not provide a specific consent. They had provided an informed consent to the clinical procedures performed as outpatients at the geriatric clinic, as per good clinical practice. The analyses were performed, and the results reported with full respect of anonymity and confidentiality. The data retrieved for the purpose of this study were not used for any other purpose.

\section{AUTHOR CONTRIBUTIONS}

GD and MM are the lead investigators and headed study design, data collection, and article writing. MM is also the senior mentor of the study and oversaw the study design. VS, AG, AA, and GF are coinvestigators and participated in study design, data collection, and article review. SD helped with statistical analysis and participated in article review. PR, CA, SI, and BA contributed to the study design, facilitated access to medical records, and participated in article review. DM is the medical director of the Geriatric Unit of the Fondazione IRCCS Ca' Granda Ospedale Maggiore Policlinico in Milan. ES is the responsible of the Alzheimer Evaluation Unit of the Neurodegenerative disease Unit of the Fondazione IRCCS Ca' Granda Ospedale Maggiore Policlinico in Milan. DM and ES are the guarantors for study compliance to ethical principles and participated in article review. All the authors reviewed and accepted the final version of the article.

\section{ACKNOWLEDGMENTS}

We thank all the staff of the outpatient clinic of the Geriatric Unit of the Fondazione IRCCS Ca' Granda Ospedale Maggiore Policlinico in Milan.

\section{SUPPLEMENTARY MATERIAL}

The Supplementary Material for this article can be found online at http://www.frontiersin.org/article/10.3389/fmed.2017.00203/ full\#supplementary-material.

4. Consensus Recommendations for the Postmortem Diagnosis of Alzheimer's Disease. The national institute on aging, and reagan institute working group on diagnostic criteria for the neuropathological assessment of Alzheimer's disease. Neurobiol Aging (1997) 18(4 Suppl):S1-2.

5. Jack CR Jr, Knopman DS, Jagust WJ, Shaw LM, Aisen PS, Weiner MW, et al. Hypothetical model of dynamic biomarkers of the Alzheimer's pathological cascade. Lancet Neurol (2010) 9(1):119-28. doi:10.1016/S14744422(09)70299-6

6. Dubois B, Feldman HH, Jacova C, Hampel H, Molinuevo JL, Blennow K, et al. Advancing research diagnostic criteria for Alzheimer's disease: the IWG-2 criteria. Lancet Neurol (2014) 13(6):614-29. doi:10.1016/S14744422(14)70090-0

7. Ossenkoppele R, Sen WJ, Rabinovici GD, Knol DL, van der Flier WM, van Berckel BN, et al. Prevalence of amyloid PET positivity in dementia syndromes: a meta-analysis. JAMA (2015) 313(19):1939-49. doi:10.1001/jama.2015.4669 
8. Jack CR Jr, Wiste HJ, Weigand SD, Therneau TM, Knopman DS, Lowe V, et al. Age-specific and sex-specific prevalence of cerebral $\beta$-amyloidosis, tauopathy, and neurodegeneration in cognitively unimpaired individuals aged 50-95 years: a cross-sectional study. Lancet Neurol (2017) 16(6):435-44. doi:10.1016/S1474-4422(17)30077-7

9. Savva GM, Wharton SB, Ince PG, Forster G, Matthews FE, Brayne C, et al. Age, neuropathology, and dementia. N Engl J Med (2009) 360(22): 2302-9. doi:10.1056/NEJMoa0806142

10. Dolan D, Troncoso J, Resnick SM, Crain BJ, Zonderman AB, O’Brien RJ. Age, Alzheimer's disease and dementia in the baltimore longitudinal study of ageing. Brain (2010) 133(Pt 8):2225-31. doi:10.1093/brain/awq141

11. Gorelick PB, Scuteri A, Black SE, Decarli C, Greenberg SM, Iadecola C, et al. American Heart Association Stroke Council, Council on Epidemiology and Prevention, Council on Cardiovascular Nursing, Council on Cardiovascular Radiology and Intervention, and Council on Cardiovascular Surgery and Anesthesia. Vascular contributions to cognitive impairment and dementia: a statement for healthcare professionals from the American Heart Association/American Stroke Association. Stroke (2011) 42(9):2672-713. doi:10.1161/STR.0b013e3182299496

12. Dubois B, Feldman HH, Jacova C, Cummings JL, Dekosky ST, Barberger-Gateau P, et al. Revising the definition of Alzheimer's disease: a new lexicon. Lancet Neurol (2010) 9(11):1118-27. doi:10.1016/S1474-4422 (10)70223-4

13. Howard R, McShane R, Lindesay J, Ritchie C, Baldwin A, Barber R, et al. Donepezil and memantine for moderate-to-severe Alzheimer's disease. N Engl J Med (2012) 366(10):893-903. doi:10.1056/NEJMoa1106668

14. Onor ML, Trevisiol M, Negro C, Aguglia E. Different perception of cognitive impairment, behavioral disturbances, and functional disabilities between persons with mild cognitive impairment and mild Alzheimer's disease and their caregivers. Am J Alzheimers Dis Other Demen (2006) 21(5):333-8. doi:10.1177/1533317506292454

15. Gauthier S, Reisberg B, Zaudig M, Petersen RC, Ritchie K, Broich K, et al. International Psychogeriatric Association Expert Conference on mild cognitive impairment. Mild cognitive impairment. Lancet (2006) 367(9518): 1262-70. doi:10.1016/S0140-6736(06)68542-5

16. Visser PJ, Verhey F, Knol DL, Scheltens P, Wahlund LO, Freund-Levi Y, et al. Prevalence and prognostic value of CSF markers of Alzheimer's disease pathology in patients with subjective cognitive impairment or mild cognitive impairment in the DESCRIPA study: a prospective cohort study. Lancet Neurol (2009) 8(7):619-27. doi:10.1016/S1474-4422(09)70139-5

17. Caffarra P, Vezzadini G, Dieci F, Zonato F, Venneri A. Rey-Osterrieth complex figure: normative values in an Italian population sample. Neurol Sci (2002) 22(6):443-7. doi:10.1007/s100720200003

18. Orsini A, Grossi D, Capitani E, Laiacona M, Papagno C, Vallar G. Verbal and spatial immediate memory span: normative data from 1355 adults and 1112 children. Ital J Neurol Sci (1987) 8(6):539-48. doi:10.1007/BF02333660

19. Folstein MF, Folstein SE, McHugh PR. "Mini-mental state". A practical method for grading the cognitive state of patients for the clinician. J Psychiatr Res (1975) 12(3):189-98. doi:10.1016/0022-3956(75)90026-6

20. Raven JC. Manuale Di Istruzioni Delle Matrici Progressive Colore. Firenze: Organizzazioni Speciali (1984).

21. Spinnler H, Tognoni G. Standardizzazione e taratura italiana di test neuropsicologici. Ital J Neurol Sci (1987) 8(Suppl 8):1-120.

22. Giovagnoli AR, Del Pesce M, Mascheroni S, Simoncelli M, Laiacona M, Capitani E. Trail making test: normative values from 287 normal adult controls. Ital J Neurol Sci (1996) 17(4):305-9. doi:10.1007/BF01997792

23. Novelli G, Papagno C, Capitani E, Laiacona M, Vallar G, Cappa SM. Three clinical tests for the assessment of lexical retrieval and production: norms from 320 normal subjects. Arch Psicol Neurol Psichiatr (1986) 47(4): 477-506.

24. Laiacona M, Barbarotto R, Trivelli C, Capitani E. Dissociazioni semantiche intercategoriali: descrizione di una batteria standar-dizzata e dati normativi. Arch Psicol Neurol Psichiatr (1993) 54(2):209-48.

25. Fazekas F, Chawluk JB, Alavi A, Hurtig HI, Zimmerman RA. MR signalabnor malities at $1.5 \mathrm{~T}$ in Alzheimer's dementia and normal aging. AJR Am J Roentgenol (1987) 149(2):351-6. doi:10.2214/ajr.149.2.351

26. Rösler M, Anand R, Cicin-Sain A, Gauthier S, Agid Y, Dal-Bianco P, et al. Efficacy and safety of rivastigmine in patients with Alzheimer's disease: international randomised controlled trial. BMJ (1999) 318(7184):633-8. Erratum in: BMJ (2001) 322(7300):1456. doi:10.1136/bmj.318.7184.633

27. Rogers SL, Farlow MR, Doody RS, Mohs R, Friedhoff LT. A 24-week, doubleblind, placebo-controlled trial of donepezil in patients with Alzheimer's disease. Donepezil Study Group. Neurology (1998) 50(1):136-45. doi:10.1212/ WNL.50.1.136

28. Wilcock GK, Lilienfeld S, Gaens E. Efficacy and safety of galantamine in patients with mild to moderate Alzheimer's disease: multicentre randomised controlled trial. Galantamine International-1 Study Group. BMJ (2000) 321(7274):1445-9. Erratum in: BMJ (2001) 322(7283):405. doi:10.1136/bmj. 321.7274.1445

29. Reisberg B, Doody R, Stöffler A, Schmitt F, Ferris S, Möbius HJ, et al. Memantine in moderate-to-severe Alzheimer's disease. N Engl J Med (2003) 348(14):1333-41. doi:10.1056/NEJMoa013128

30. Abbate C, Trimarchi PD, Nicolini P, Bergamaschini L, Vergani C, Mari D. Comparison of informant reports and neuropsychological assessment in mild cognitive impairment. Am JAlzheimers Dis Other Demen (2011) 26(7):528-34. doi:10.1177/1533317511429323

31. Jansen WJ, Handels RL, Visser PJ, Aalten P, Bouwman F, Claassen J, et al. The diagnostic and prognostic value of neuropsychological assessment in memory clinic patients. J Alzheimers Dis (2017) 55(2):679-89. doi:10.3233/ JAD-160126

32. Meijs AP, Claassen JA, Rikkert MG, Schalk BW, Meulenbroek O, Kessels RP, et al. How does additional diagnostic testing influence the initial diagnosis in patients with cognitive complaints in a memory clinic setting? Age Ageing (2015) 44(1):72-7. doi:10.1093/ageing/afu053

33. Mattsson N, Rosén E, Hansson O, Andreasen N, Parnetti L, Jonsson M, et al. Age and diagnostic performance of Alzheimer disease CSF biomarkers. Neurology (2012) 78(7):468-76. doi:10.1212/WNL.0b013e3182477eed

34. Wardlaw JM, Smith EE, Biessels GJ, Cordonnier C, Fazekas F, Frayne R, et al. Standards for ReportIng Vascular changes on neuroimaging (STRIVE v1). Neuroimaging standards for research into small vessel disease and its contribution to ageing and neurodegeneration. Lancet Neurol (2013) 12(8): 822-38. doi:10.1016/S1474-4422(13)70124-8

Conflict of Interest Statement: The authors declare that the research was conducted in the absence of any commercial or financial relationships that could be construed as a potential conflict of interest.

Copyright (c) 2017 Dolci, Damanti, Scortichini, Galli, Rossi, Abbate, Arosio, Mari, Arighi, Fumagalli, Scarpini, Inglese and Marcucci. This is an open-access article distributed under the terms of the Creative Commons Attribution License (CC BY). The use, distribution or reproduction in other forums is permitted, provided the original author(s) or licensor are credited and that the original publication in this journal is cited, in accordance with accepted academic practice. No use, distribution or reproduction is permitted which does not comply with these terms. 